\title{
Sufficient Conditions for Hamiltonicity in Communication Networks through Graph Modeling: New Research Challenges
}

\author{
Ajeet Singh \\ Research Fellow \\ CR Rao AIMSCS \\ UOH Campus, Hyderabad-500046, India
}

\author{
Vikas Tiwari \\ Research Fellow \\ CR Rao AIMSCS \\ UOH Campus, Hyderabad-500046, India
}

\begin{abstract}
Graphs can be used to model various problems and relations in diverse domains eg. Computer Science, Biological, Chemical and many other. There exist various hard problems in communication networks, which are practically hard but can be shaped in the form of graphs. A Hamiltonian Path is a spanning trail in a network graph i.e. a path through every network node and a spanning cycle in a network graph is a Hamiltonian cycle. A network graph containing a Hamiltonian Cycle in it, is said to be Hamiltonian. The problem of finding whether a graph $G$ is Hamiltonian is proved to be NPComplete. It cann't be solved in a polynomial time.

In this paper, state-of-the-art for existing degree related conditions for graphs to posses Hamiltonian cycle is presented. Later, various practical applications of Graph Theory in computer science are summarized. Future research directions in this perspective are also given at the end of this paper.
\end{abstract}

\section{Keywords}

Graphs, Communication networks, Hamiltonian cycles, Spanning cycle, Hamiltonian path.

\section{INTRODUCTION}

Today, many hard problems of practical interest need to be solved. One of the challenging area is, communication networks and packet routing problems. In order to solve these hard problems, network can be modeled as a graph, in which network nodes can be thought of as vertices of graph and communication path inside the network can be represented as edges in the graph. Graph Theory can be thought of as such an efficient tool to model these practical problems with the help of Graphs. Graph Theory approach has its wide applications in diverse fields. [2][6] Some well known problems in Computer Science like- Travelling salesman problem [11], whose objective is to start from a city and find out the shortest possible path, visiting all cities and return back to the same point. This problem can also be thought of in the perspective of communication networks system and can be well formulated in Graphs, where nodes or vertices of the graph represent the cities and path is represented by edges inside the graph. In this way, a model can be made for a particular practical problem.

A network graph can be thought as a finite set of network nodes, connected by path links which are called as edges, when a network is modeled in the form of graph. A Graph can be directed or undirected. If the edges of the graph are associated with directions, then it is called directed graph(digraph) and if the edges don't have it's directions, then it is called undirected graph. A graph is shown as figure 1 below:-

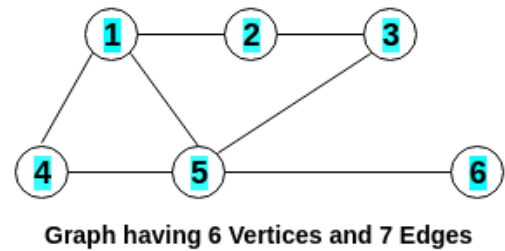

Fig. 1

Some other open hard problems in network graphs [17] are summarized as below:-

(1) Finding bipartite set in a network graph

(2) Finding independant set in a graph

(3) Searching edge domination in a graph

(4) Finding minimum cardinality domination set

(5) Finding independant domination set

(6) Finding dissociation set and matching number

(7) Finding Hamiltonian cycle

(8) Finding cycle of maximum length in a network graph

(9) Exploring longest path

(10) Vertex and edge coloring problem

A Hamiltonian Path is a path through every vertex. A Hamiltonian cycle is a spanning cycle in the graph and a graph containing a Hamiltonian Cycle is said to be Hamiltonian.

Famous Irish mathematician Sir William Rowan Hamilton, in 1859 first posed hamiltonian Circuit Problem, is still unsolved. It is an NP-Complete problem. It is clear that every graph with a Hamiltonian Cycle has a Hamiltonian path but the converse is not necessarily true. 


\subsection{Motivation to the problem}

In communication networks, while designing any routing protocol, there may occur some practically hard problems, whose solution may possible by modeling them in form of graphs. Hamiltonicity is the property of any network graph to test weather Hamiltonian cycle exist or not. Any graph is supposed to be Hamiltonian if it consists a Hamiltonian Cycle. The problem of finding whether a network graph $G$ is Hamiltonian is proved to be NP-Complete. It cann't be solved in a polynomial time.

Tremendous amount of research has been done over past years and various degree related conditions for graphs to posses Hamiltonian cycle have been explored. Many sufficient conditions exist in literature to test the hamiltonicity of network graphs. Still good amount of research has to be done to explore more the computationally efficient constraints for presence of hamiltonicity in network structured graphs.

\subsection{Organization of the paper}

The general structure of rest of this paper is as, Section 2 presents some preliminaries required. Section 3 presents the overview of related work. Section 4 explains some of the wide range applications of Graph Theory on Computer Science. In section 5, future research directions in this perspective are presented. Finally, section 6 concludes the paper.

\section{PRELIMINARIES}

Some basic preliminaries required in this paper are described as below:-

\subsection{Undirected graph}

Consider, a graph $G=(V, E)$ given as figure 2 below -

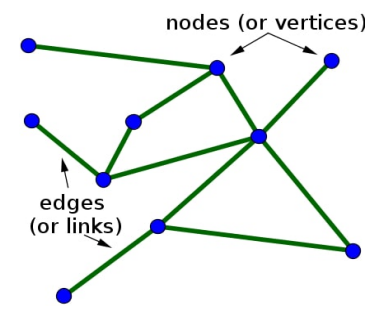

Fig. 2

Here, $V$ : finite set of network nodes and $E$ : finite set of edges. An undirected graph is a graph in which existing objects or network nodes are connected through bi-directional links.

\subsection{Directed graph}

Consider, a graph $G=(V, E)$ given as figure 3 below -

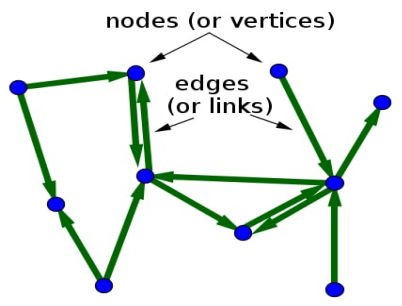

Fig. 3

Here, $V$ : finite set of network nodes and $E$ : finite set of edges. A directed graph or digraph is a graph in which existing objects or network nodes are connected to each other through uni-directional links.

\subsection{Walk and Path}

Suppose, $V=\left(v_{0}, v_{1}, v_{2}, \ldots, v_{n}\right)$ be any specific sequence of vertices, then -

$V$ is a Walk, if all edges in $V$ are distinct and it is a path if all vertices in $V$ are distinct.

\subsection{Trees}

If there is a connected graph with presence of no cycles in it, then it is considered as a tree.

For any tree if $V$ is the set of network nodes or vertices and $E$ is the set of edges. Then $|E|=\{|V|-1\}$

\subsection{Weighted graph}

If edges in the graph $G=(V, E)$ are associated with it's corresponding weights, it is termed as a weighted graph. In practical networks scenario, these edge weights can be thought of as path distances.

\subsection{Multigraph}

A graph $G=(V, E)$ is a kind of network graph in which multiple edges are possible between two vertices.

\subsection{Isolated vertex}

In a graph $G=(V, E)$, the vertex in which no edges are incident on it, is called as isolated vertex.

\subsection{Adjacency matrix}

An adjacency matrix can be represented as an $n \times n$ matrix $A=a_{i j}$ for a graph with vertex set Vert = $\left\{\right.$ vert $_{1}$, vert $_{2}$, vert $_{3}, \ldots$, vert $\left._{n}\right\}$, where $a_{i j}$ is the number of edges which join vert $_{i}$ and vert $_{j}$.

\subsection{Bipartite graph}

If there is a possibility to divide the vertices of a network graph $G=(V, E)$ into two sets such that each edge or path is having it's one end at one set and other edge end belongs to some other vertex set, then that kind of network graph structute is considered as a bipartite graph. A bipartite graph is shown as figure 4 below:-

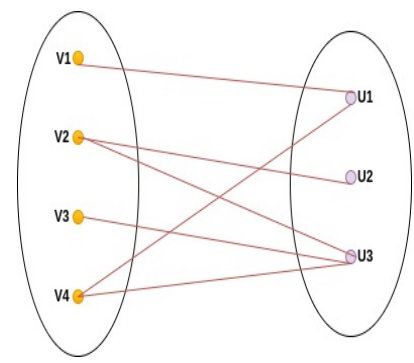

Fig. 4 


\subsection{Markov random field}

Markov random network, a kind of graphical model consists a set of random variables, possesing the Morkov property described by an undirected graph. Markov random network can be acyclic as well as undirected. Markov random network is somewhat similar to Bayesian networks.

\section{RELATED WORK}

The research of Hamiltonian Cycles and Hamiltonian paths in general as well as in special graphs has been fired by the practical applications and by issues of the complexity. Neither efficiently testable condition is known for Hamiltonian graphs nor there exists any such condition to test whether a graph consists of a Hamiltonian path or not. This is the prime reason, why tremendous amount of research has been done in the past for finding the sufficient and adequate conditions for the existence of Hamiltonian Cycles or Hamiltonian paths in graphs.

We will present here some degree related conditions on which the previous research has been done. [3]|7]|[8]|9]|[10] Some of the notations and definitions, we will use in this paper are as follows:-

Consider a graph $G=(V, E)$,

where -

$V(G)$ : finite set of nodes/vertices

$E(G)$ : finite set of edges

Let, a vertex $u \in V$. By $d(u)$ we mean, the degree of $u$ in $G$. $\delta(G)$ indicates the degree of a minimum degree vertex in graph $G$.

Different degree related conditions, which have been proposed in past years for graphs to posses Hamiltonian cycle are presented below:-

TheOrem 1 DiraC's THEOREM. Consider, $G$ is a simple graph with $n$ vertices.

where $n \geq 3$ and $\delta(G) \geq \frac{n}{2}$, then graph $G$ is Hamiltonian.

THEOREM 2 ORE's THEOREM. Consider, $G$ is a simple graph with $n$ vertices and $u, v$ be distinct nonadjacent vertices of $G$ with $d(u)+d(v) \geq n$, then graph $G$ is Hamiltonian.

Theorem 3 Bondy-ChVtal's theorem. Consider, $G$ is a simple graph with $n$ vertices, then $G$ is Hamiltonian if and only if its closure is Hamiltonian.

Theorem 4. Consider, $G=(V, E)$ is Hamiltonian and if $S$ is any non-empty proper subset of $V$, the graph $G-S$ has at most $|S|$ components.

THEOREM 5 MEYNIEL'S THEOREM. A strongly connected digraph with $n$ vertices is Hamiltonian if the sum of the degrees of every pair of nonadjacent vertices is at least $(2 n-1)$.

THEOREM 6 WOODALL'S THEOREM. A nontrivial digraph with $n$ vertices is Hamiltonian if (outdegreeofu) + (indegreeofv) $\geq n$, whenever $u$ and $v$ are vertices such that there is no arc from vertex $u$ to vertex $v$.

Theorem 7 GHOUla-Houri THEOREM. A strongly connected digraph with $n$ vertices is Hamiltonian if the degree of each vertex is at least $n$.

THEOREM 8. A directed graph with $n$ vertices is Hamiltonian, if both the indegree and outdegree of each vertex are at least $\frac{n}{2}$.

THEOREM 9. If a graph $G$ contain a pendant edge, then Hamiltonian Cycle will not exist.
In [1], a new proposed sufficient condition is given for a graph to contain Hamiltonicity property. Suppose $G$ is a graph having $n$ finite vertices and $e$ edges. If degree $(u)+$ degree $(v)+\delta(u, v)$ is at least $(n+1)$, where $u$ and $v$ are the distinct non-adjacent vertices in the graph $G$. Then this kind of graph satisfying above condition will be having Hamiltonian Path.

Gerald L. Thompson et al.[4] have proposed an efficient algorithm to discover Hamiltonian Path in an undirected graph. They tested this algorithm for graph containing upto 1000 vertices.

In 2009, Jordan Baumgardner et al.[5] have proposed a mechanism to solve Hamiltonian circuit problem by using bacterial computer. Bacterium DNA computers have been designed to make a try for dealing with this Hamiltonian circuit problem, which is proved to be NP complete. This biological principle has been tested successfully and approximated for discovering a Hamiltonian Path in a graph with 3 vertices. This inspired the researchers upto some extent. In 2000, Teunter et. al. [12] presented the lower and upper bound result conditions for hamiltonicity in network graphs. Wagner et. al. [13] presented an ant inspired heuristic model for hamiltonian graphs detection. Zhu et. al. [14] presented some conditions for hamiltonian based on linear diophantine equation systems with cycle vector.

In 2010, Lizhi Du [15] presented a polynomial time algorithm for hamiltonian cycle. Drawback of this mechanism was, it is computationally much high for large sized network graphs. Later, Stewart [16] proposed sufficient conditions for hamiltonicity in multiswapped network structures.

\section{WIDE RANGE APPLICATIONS OF GRAPH THEORY IN COMPUTER SCIENCE}

Various research problems in the domain of Computer Science are modeled into the form of graph algorithms. For example route problems in networking are modeled in the form of finding shortest path between two vertices in a graph. Some of the widely-known problematic algorithms are presented below :-

(1) Algorithm to find shortest path among nodes inside a network.

(2) Algorithm to find out planarity and non-planarity of a graph.

(3) Finding MST(minimum spanning tree) algorithm.

(4) Adjacency list and Adjacency matrices finding algorithms.

(5) Algorithm to find connected components in a network structure.

(6) Algorithm to discover Eulerian Circuit, Hamiltonian Path and Hamiltonian circuit in a given graph.

(7) Algorithm to search for an element using Depth First Search and Breadth-First Search.

(8) Algorithm for Travelling Salesman Problem(TSP).

(9) Routing Algorithms to discover network flow.

(10) Graph Coloring Algorithm

Above applications are presented to project and demonstrate the significance of graph theory in computer science.

\section{RESEARCH DIRECTIONS}

Graph theory modeling has been employed with much success for solving hard problems inside communication networks. Exploring the Hamiltonicity inside network graphs is still unsolved over long time and proved to be NP-Complete problem. Still this problem has attracted many researchers from all over the world and development is going on in this area. Our future research directions are summarized as below - 


\subsection{Future Research Directions}

Discovering Hamiltonian circuit in a graph is well proved to be NP-Complete problem. The sufficient conditions presented above in this paper are previously existing degree related conditions. All these conditions are proved to be tight.

We will explore more the condition of pairwise non-adjacent vertex triplets for Hamiltonian Path and later for Hamiltonian Cycles. The extention to this research may be the conditional approach of vertex-quadruples and more and in further instance hypothesize the cases.

\section{CONCLUSION}

Wide variety problems of Computer Science and many other fields can be modeled in the form of Graphs. Graph algorithms can be applied to solve these problems. In this paper, the various degree related conditions for graphs to posses Hamiltonian cycle are presented, which have been proposed in past years. Wide range of practical and real time networking applications of Graph Theory in various engineering, biological and other domains are presented in the paper. An overview of future research directions and emerging scenarios are also given.

\section{REFERENCES}

[1] Rao Li, "A new sufficient condition for Hamiltonicity of graphs", Elsevier, Information Processing Letters 98 (2006) 159161.

[2] Ferozuddin Riaz and Ferozuddin Riaz, "Applications of Graph Theory in Computer Science”, 2011 IEEE Third International Conference on Computational Intelligence, Communication Systems and Networks.

[3] Ali Eshragh, "Can Hamiltonian Cycle Problem be Solved with High Probability in Polynomial Time?", School of Mathematical Sciences, The University of Adelaide, Adelaide SA 5005 Australia, February, 2012.

[4] Gerald L. Thompson, Sharad Singhal, "A Successful Algorithm For The Undirected Hamiltonian Path Problem", Carnegie-Mellon University Pittsburgh, 1984.

[5] Jordan Baumgardner, Karen Acker et. al., "Solving a Hamiltonian Path Problem with a bacterial computer", Journal of Biological Engineering, 2009.

[6] J.A. Bondy, U.S.R. Murty, "Graph Theory with Applications", Macmillan/Elsevier, London/New York, 1976.

[7] O. Ore, Note on Hamiltonian Circuits.

[8] N. Deo, "Graph Theory: With applications to engineering and Computer Science", PHI, Eastern Economy Edition.

[9] D.B. West, "Introduction to Graph Theory", Prentice-Hall, Inc., New Jersey.

[10] M.R. Garey and D.S. Jhonson, "Computers and Intractability: A Guide to the Theory of NP Completeness", W.H. Freeman and Company.

[11] K. Rosen, Discrete Mathematics and its Application, 6th Ed., McGraw-Hill.

[12] Ruud H. Teunter, Edo S. van der Poort, "The maximum number of Hamiltonian cycles in graphs with a xed number of vertices and edges", Operations Research Letters 26 (2000) 91-98.

[13] Israel A. Wagner, Alfred M. Bruckstein, "Hamiltonian(t) - An Ant-Inspired Heuristic for Recognizing Hamiltonian Graphs", (1999) IEEE.
[14] Guohun Zhu, Chunwei Song, Kaoru Hirota, F angyan Dong, $\mathrm{Y}$ onghua Wu, "Necessary and Sufficient Conditions for Hamiltonian based on Linear Diophantine Equation Systems with Cycle Vector", 2009 Third International Conference on Genetic and Evolutionary Computing.

[15] Lizhi Du, "A Polynomial Time Algorithm for Hamilton Cycle (Path)", intern. multi-conference of engineers and computer scientists, Mar 17-19, (2010), HongKong.

[16] Iain A. Stewart, "Sufficient conditions for Hamiltonicity in multiswapped networks", J. Parallel Distrib. Comput. 101 (2017) 1726

[17] V.E. Alekseev, R. Boliac, D.V. Korobitsyn, V.V. Lozin, "NPhard graph problems and boundary classes of graphs", Theoretical Computer Science 389 (2007) 219-236, ELSEVIER. 